\title{
Incidence angle dependence of Langmuir turbulence and artificial ionospheric layers driven by high-power HF-heating
}

\author{
B. Eliasson ${ }^{1,2}$, G. Milikh ${ }^{1}$, X. Shao ${ }^{1}$, E. V. Mishin ${ }^{3}$, and K. Papadopoulos ${ }^{1}$ \\ ${ }^{1}$ Departments of Physics and Astronomy, University of Maryland, College Park, \\ Maryland, USA. \\ ${ }^{2}$ Physics Department, University of Strathclyde, Glasgow, Scotland, UK. \\ ${ }^{3}$ Space Vehicles Directorate, Air Force Research Laboratory, Kirtland AFB,
} Albuquerque, NM, USA.

\begin{abstract}
:
We have numerically investigated the development of strong Langmuir turbulence (SLT) and associated electron acceleration at different angles of incidence of ordinary $(\mathrm{O})$ mode pump waves. For angles of incidence within the Spitze cone, the turbulence initially develops within the first maximum of the Airy pattern near the plasma resonance altitude. After a few milliseconds, the turbulent layer shifts downwards by about one kilometer. For injections outside the Spitze region, the turning point of the pump wave is at lower altitudes. Yet, an Airy-like pattern forms here, and the turbulence development is quite similar to that for injections within the Spitze. SLT leads to the acceleration of $10-20 \mathrm{eV}$ electrons that ionize the neutral gas thereby creating artificial ionospheric layers. Our numerical modeling shows that most efficient electron acceleration and ionization occur at angles between the magnetic and geographic zenith, where SLT dominates over weak turbulence. Possible effects of the focusing of the electromagnetic beam on magnetic field-aligned density irregularities and the finite heating beam width at the magnetic zenith are also discussed. The results have relevance to ionospheric heating experiments using ground-based, high-power radio transmitters to heat the overhead plasma, where recent observations of artificial ionization layers have been made.
\end{abstract}

PACS: 94.20.Tt, 94.20.Bb, 94.20.wj, 94.20.ws, 94.20.dv

\section{Introduction}

Recently, there have been a number of observations of artificial ionospheric layers created by high-power ordinary radio waves injected into the F-region ionospheric plasma layer. These observations were made at the High-frequency Active Auroral Program (HAARP) facility after its update to 3.6 MW, and were not seen at the previous 
960 kW level (Pedersen et al. 2009; 2010; Mishin and Pedersen 2011; Sergeev et al. 2013). Once the artificial plasma reached sufficient density to support interaction with the transmitter beam, it rapidly descended as an ionization wave from about 220 to $\sim 150 \mathrm{~km}$ altitude. The formation of ionospheric layers is attributed to the acceleration of electrons by turbulence at the critical height of the $\mathrm{O}$ mode wave (Mishin and Pedersen, 2011). To study the processes leading to the formation of descending artificial ionospheric layers (DAILs), a numerical study was carried out with a one-dimensional geometry along the vertical line using typical HAARP parameters (Eliasson et al. 2012). As it follows from this study, SLT develops just below the turning point of the $\mathrm{O}$ mode wave in a layer with altitude extent of a few hundred meters and provides efficient electron acceleration. DAILs have been simulated using a Fokker-Planck model for the acceleration of electrons by SLT, a kinetic model for the transport of energetic electrons through the ionosphere, and a chemical dynamic model for the relevant ion species. The simulation results reproduce the salient observational features of ionization fronts descending from about $230 \mathrm{~km}$ to $150 \mathrm{~km}$ in a few minutes. While the simulation studies of Eliasson et al. (2012) was carried out for vertical incidence of the electromagnetic wave, the experiments (Pedersen et al. 2010; Mishin and Pedersen, 2011; Sergeev et al. 2013) were conducted with the HF radio beam centered at the magnetic zenith, which is 14.5 degrees south to the vertical at HAARP. Early experiments have also shown enhanced electron heating and incoherent radar backscatter near MZ (e.g., Kosch et al. 2000; Pedersen et al. 2003; Rietveld et al. 2003). Gurevich et al. (2002) suggested that this MZ effect is determined by self-focusing of the electromagnetic beam on magnetic field-aligned striations that develop on time scales of several seconds and are associated with upper hybrid turbulence well below the turning point within the Spitze. There is significant anomalous absorption of the electromagnetic wave on striations, which usually inhibits the SLT development near the plasma resonance. Honary et al. (2011) have found that while the electron temperature at $\mathrm{MZ}$ increases to a value about twice of that for vertical incidence, the most efficient electron heating is at some intermediate angle between the $\mathrm{MZ}$ and Spitze. Using a low duty cycle $(0.2 \mathrm{~s}$ every $10 \mathrm{~s})$ of the high-frequency transmitter to excite Langmuir turbulence, Isham et al. (1999) reported radar observations of plasma waves that were strongest when the radar beam was directed between the Spitze angle and MZ, and that the observed ion fluctuations could occur several kilometers below the critical height outside the Spitze region. The aim of this paper is to study how different angles of incidence influence the SLT and the formation of DAILs. In doing so, we extend the previously developed numerical model for vertical incidence (Eliasson et al. 2012; Eliasson 2013) to take into account oblique angles of incidence.

\section{Full-wave modeling of strong Langmuir turbulence}

A generalized Zakharov model (Eliasson 2013) is employed to simulate the propagation of the high-frequency $\mathrm{O}$ mode electromagnetic wave through ionosphere, and its conversion to short-scale, high-frequency Langmuir waves and low-frequency ion fluctuations. The mathematical model, which is an extension of the original model of Zakharov (1972) to include the electromagnetic wave and magnetic field effects, is given in the in Equations (21)-(33) of Eliasson (2013). The ionospheric density profile is assumed to be of the form $n_{i 0}(z)=n_{0, \max } \exp \left[-\left(z-z_{\max }\right)^{2} / L_{n 0}^{2}\right]$, where $n_{0, \max }=$ 
$1.436 \times 10^{11} \mathrm{~m}^{-3}$ is the electron density at the $\mathrm{F}_{2}$ peak located at $z_{\max }=242 \mathrm{~km}$, and $L_{n 0}=31.62 \mathrm{~km}$ is the ionospheric scale length. The $\mathrm{F}_{2}$-peak electron density corresponds to the peak plasma frequency $f_{O F_{2}}=3.40 \mathrm{MHz}$, while the pump frequency is taken $f_{0}=3.20 \mathrm{MHz}\left(\omega=20.1 \times 10^{6} \mathrm{~s}^{-1}\right)$. For the chosen parameters, the critical density $n_{i 0}=n_{c}=1.27 \times 10^{5} \mathrm{~cm}^{-3}$ where the pump frequency equals the plasma frequency is at $z_{O} \approx 230.96 \mathrm{~km}$. At the critical altitude, the local plasma length scale is $L_{n}=1 /\left.\left(d \ln n_{0} / d z\right)\right|_{z_{0}}=43 \mathrm{~km}$. The simulations use an injected $\mathrm{O}$ mode with the amplitude $1 \mathrm{~V} / \mathrm{m}$, and different angles of incidence. The magnetic field of $B_{0}=5.17 \times$ $10^{-5} \mathrm{~T}$ is directed downwards $14.5^{\circ}$ to the vertical line, and gives the electron gyro frequency $1.45 \mathrm{MHz}\left(\omega_{c e}=9.09 \times 10^{6} \mathrm{~s}^{-1}\right)$. The electron and ion temperatures are taken to be $T_{e}=0.4 \mathrm{eV}$ and $T_{i}=0.2 \mathrm{eV}$, which is about two times higher than in the quiet ionosphere. The enhanced $T_{e}$ near the critical layer can be produced jointly by heating of ionospheric electrons directly by SLT and via fast electron thermal conduction from the upper hybrid layer [cf. Pedersen et al., 2010]. It was found in Eliasson et al. (2012) that $T_{e} \gtrsim 0.4 \mathrm{eV}$ was needed to efficiently accelerate the tail electrons by SLT to produce DAILs, while direct ionization via bulk heating would require a much higher electron temperature of $T_{e} \gtrsim 1.5 \mathrm{eV}$. The computational box for the $\mathrm{O}$ mode propagation extends from $z=142 \mathrm{~km}$ on the bottom side to $342 \mathrm{~km}$ on the topside ionosphere. To resolve the small-scale electrostatic waves in the turbulent region, we use a nested grid method (Eliasson, 2013), where the electromagnetic wave is resolved with a uniform grid of $4 \mathrm{~m}$, and the electrostatic turbulence and particle dynamics is resolved locally with a 4 $\mathrm{cm}$ grid in a $3 \mathrm{~km}$ wide region $z=228.5-231.5 \mathrm{~km}$ on the bottom side and $z=$ $252.5-255.5 \mathrm{~km}$ on the symmetric side at the topside ionosphere. The simulation model of Eliasson et al. (2012) and Eliasson (2013) is extended to enable oblique incidence of the $\mathrm{O}$ mode wave. To introduce an oblique angle of incidence, the envelope of the high-frequency field is assumed to have a fixed wave vector component in the $x$ direction, given by $k_{x}=k_{0} \sin \chi$, where $k_{0}=\omega / c$ is the wavenumber and $\chi$ is the angle of incidence of the injected $\mathrm{O}$ mode wave. This assumption is justified for vertical stratification of the ionospheric profile and by assuming that the slow variations in the density fluctuations associated with SLT only occur in the vertical direction. Hence, for the high-frequency field, we use $\nabla=\hat{\mathbf{x}} i k_{x}+\hat{\mathbf{z}} \partial / \partial z$. Vertical incidence (Eliasson et al. 2012) corresponds to $\chi=0^{\circ}$ and $k_{x}=0$. It should be noted that the impact of different angles of incidence on Langmuir turbulence has also been studied by Mjølhus, Helmersen and DuBois (2003) using a combination of ray-tracing and local turbulence simulations by means of a two-dimensional damped, driven Zakharov system. 

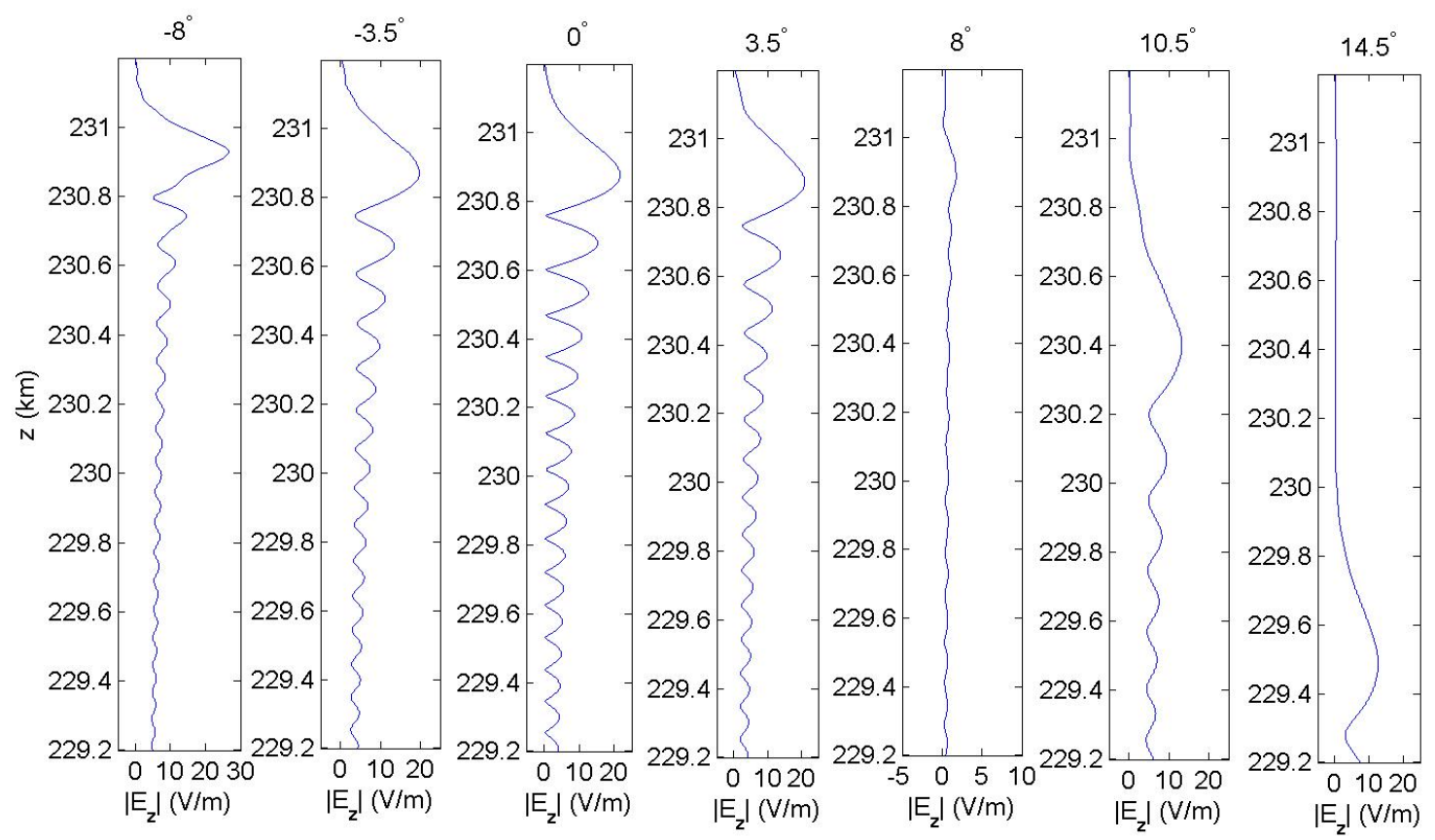

Figure 1: The amplitude of the vertical electric field at $t=1 \mathrm{~ms}$ for different angles of incidence. The cases $\chi= \pm 8^{\circ}$ corresponds to the Spitze angles where the $\mathrm{O}$ mode waves are mode converted. The $\mathrm{O}$ mode critical layer is at $z=z_{O}=230.96 \mathrm{~km}$.

Figure 1 shows the amplitude of the vertical electric field $E_{z}$ for different angles of incidence at $t=1 \mathrm{~ms}$, before any parametric instability has had time to develop. A standing wave pattern has been set up below the critical point of the $\mathrm{O}$ mode at $z=z_{O}=$ $230.96 \mathrm{~km}$, where $\omega=\omega_{p e}$. It is illustrative to compare the results in Fig. 1 with raytracing results shown in Figs. 2 and 3 for the same set of parameters as in Fig. 1. The rays of the $\mathrm{O}$ mode are obtained from the ray-tracing equations (e.g. Whitham 1974)

$$
\begin{aligned}
& \frac{d \mathbf{k}}{d t}=-\nabla \omega \\
& \frac{d \mathbf{r}}{d t}=\nabla_{\mathbf{k}} \omega
\end{aligned}
$$

using the wave frequency $\omega(\mathbf{k}, \mathbf{r})$ governed by the Appleton-Hartree dispersion relation (e.g. Stix 1992)

$$
\frac{k^{2} c^{2}}{\omega^{2}}=1-\frac{2 \omega_{p e}^{2}\left(\omega^{2}-\omega_{p e}^{2}\right) \omega^{-2}}{2\left(\omega^{2}-\omega_{p e}^{2}\right)-\omega_{c e}^{2} \sin ^{2} \theta+\omega_{c e} \Delta}
$$

where $\Delta=\left[\omega_{c e}^{2} \sin ^{4} \theta+4 \omega^{-2}\left(\omega^{2}-\omega_{p e}^{2}\right)^{2} \cos ^{2} \theta\right]^{1 / 2}$, and $\theta$ is the angle between $\mathbf{k}$ and $\mathbf{B}_{0}$. The electron plasma frequency $\omega_{p e}=\left(e^{2} n_{i 0} / \epsilon_{0} m_{e}\right)^{1 / 2}$ varies vertically with the ionospheric density profile. Figure 2 shows an overview of the large-scale ray 
propagation of the $\mathrm{O}$ mode, while a close-up of the region $z=229.2-231.2 \mathrm{~km}$ is shown in Figure 3. As seen in Figures 1 and 3, the $\mathrm{O}$ mode reaches the critical layer for $\chi=-3.5^{\circ}, 0^{\circ}$, and $3.5^{\circ}$. For $\chi=10.5^{\circ}$ and $14.5^{\circ}$, the position of the first maximum is shifted down in space about 0.3 and $1.5 \mathrm{~km}$, respectively, due to that the $\mathrm{O}$ mode is turning at lower altitudes for angles of incidence larger than the Spitze angle (e.g. Mjolhus 1990) $\chi_{c}=\arcsin [\sqrt{Y /(1+Y)} \sin (\theta)] \approx 8.04^{\circ}$ where $Y=\omega_{c e} / \omega \approx 0.45$. Ray-tracing results for EISCAT parameters (Mjolhus et al. 2003) show very similar features as here. Inside the Spitze range, the polarization of the electric field tend to be along the magnetic field as the wave reaches the critical layer, while outside the Spitze, the polarization instead approaches vertical (Mjølhus et al. 2003). When injected at the Spitze angle $\chi=\chi_{c}$, the $\mathrm{O}$ mode propagates parallel to the magnetic field as a circularly polarized $\mathrm{L}$ mode wave as it reaches the critical layer, and can then propagate into denser plasma at higher altitudes as a $\mathrm{Z}$ mode wave. The $\mathrm{F} 2$ peak has a plasma density below the critical density of the $\mathrm{Z}$ mode wave, so that $\omega_{p e}<\left(\omega^{2}+\omega \omega_{c e}\right)^{1 / 2}$, and the $\mathrm{Z}$ mode wave can therefore propagate to the conjugate point at the topside where it is again transformed to an $\mathrm{O}$ mode wave which escapes the plasma layer. Hence, for our parameters the ionosphere appears to be transparent for the $\mathrm{O}$ mode at the Spitze angle. At $\chi=-\chi_{c}$, the $\mathrm{O}$ mode is instead absorbed and transformed to a $\mathrm{Z}$ mode wave propagating down towards lower altitudes into thinner plasma until it reaches the electrostatic (Langmuir wave) resonance at $230.26 \mathrm{~km}$ where $\omega_{p e}^{2}=\omega^{2}\left(\omega^{2}-\right.$ $\left.\omega_{c e}^{2}\right) /\left(\omega^{2}-\omega_{c e}^{2} \cos ^{2} \theta\right)$. 


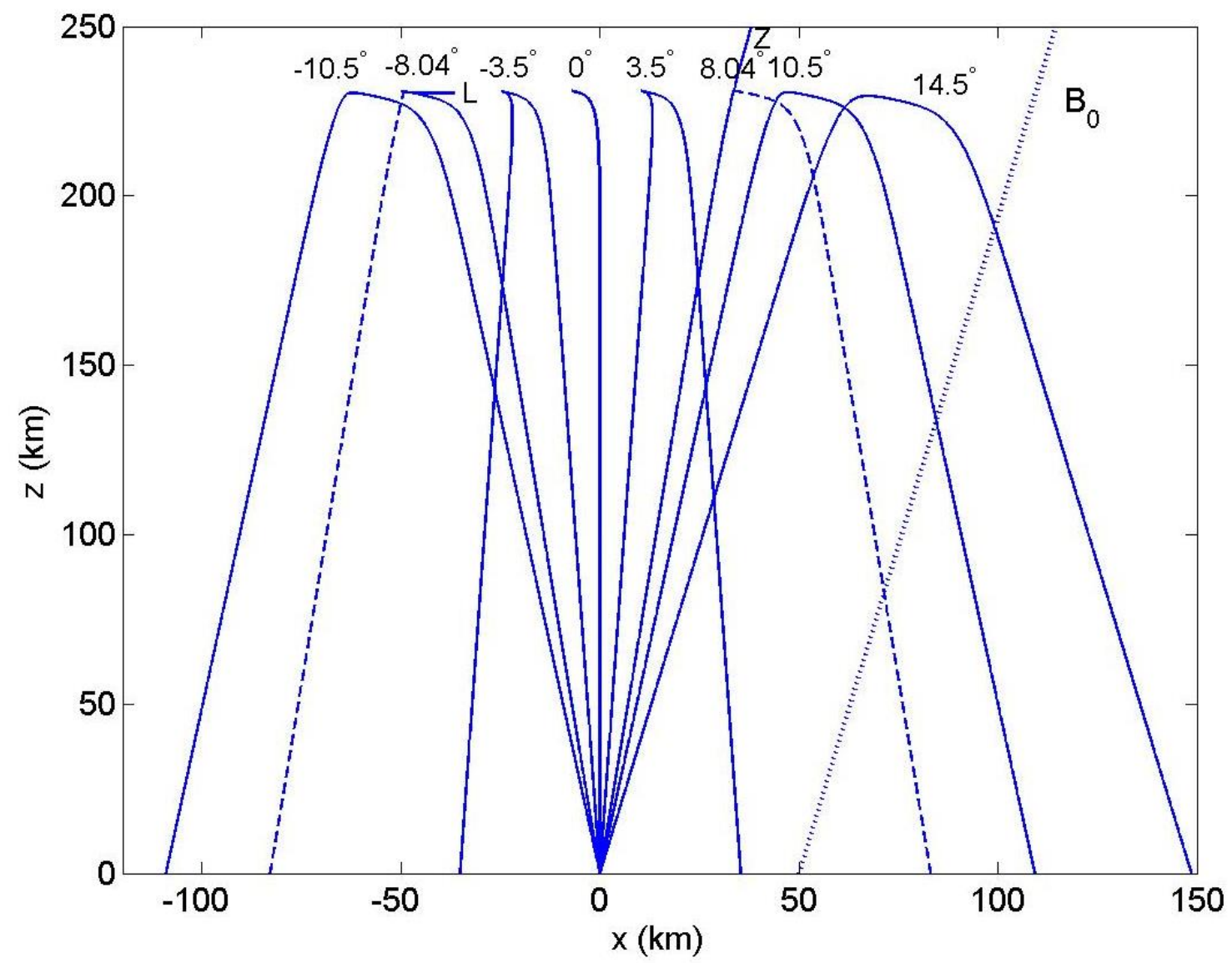

Figure 2: Ray-tracing results for $\mathrm{O}$ mode waves at different angles of incidence. The rays converted to $Z$ and Langmuir modes at the Spitze angles are indicated by ' $Z$ ', and ' $L$ ', respectively. The geomagnetic field $\mathbf{B}_{0}$ is indicated by a dotted line. 


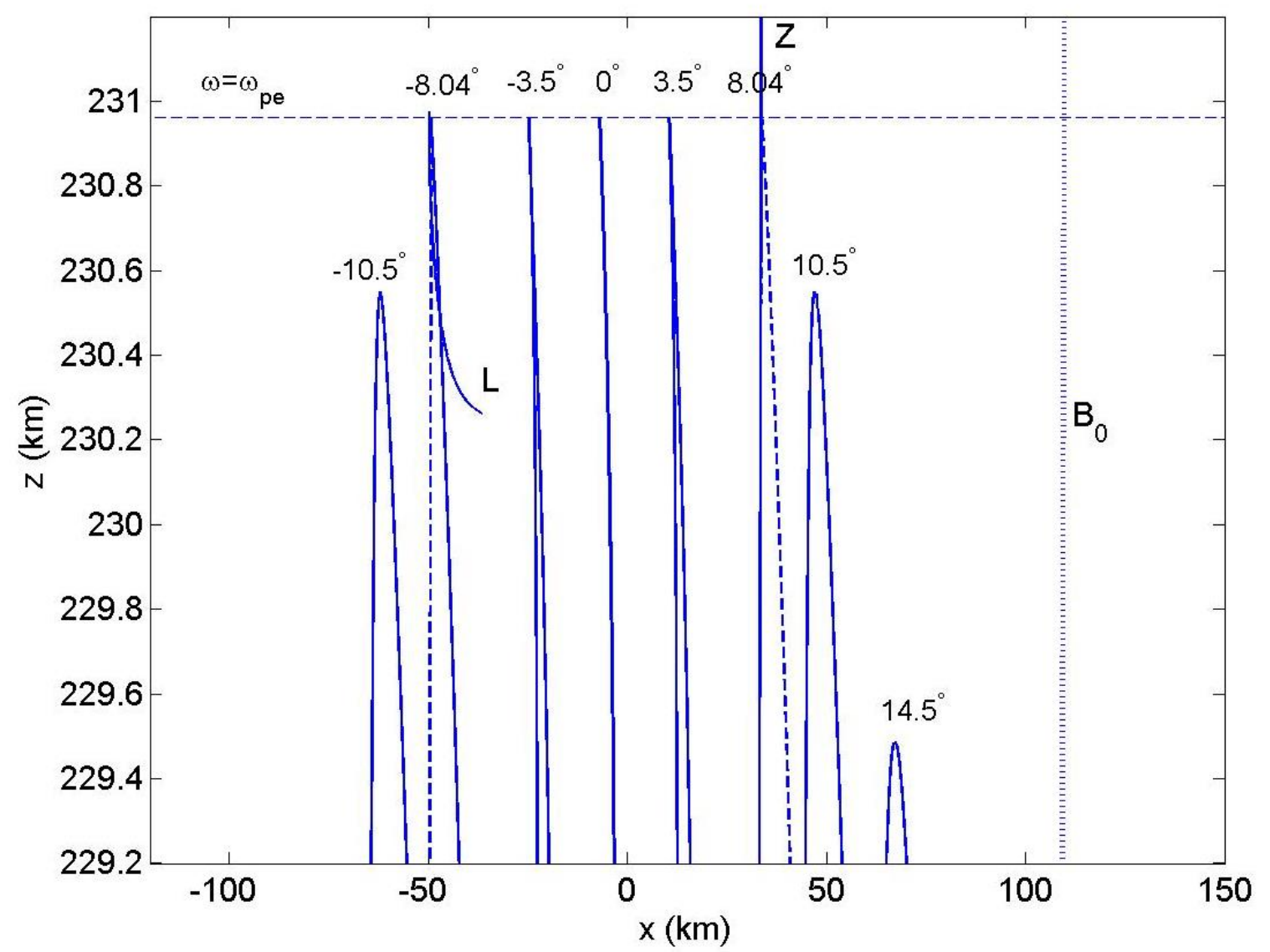

Figure 3: Closeup of the region $z=229.2-231.2 \mathrm{~km}$ in Figure 2. Rays within the Spitze region $\pm 8.04^{\circ}$ reach the critical layer $\omega=\omega_{p e}$ at $z=z_{O}=230.96 \mathrm{~km}$ (indicated with a horizontal, dashed line). Rays outside the Spitze region are reflected at lower altitudes, consistent with Figure 1.

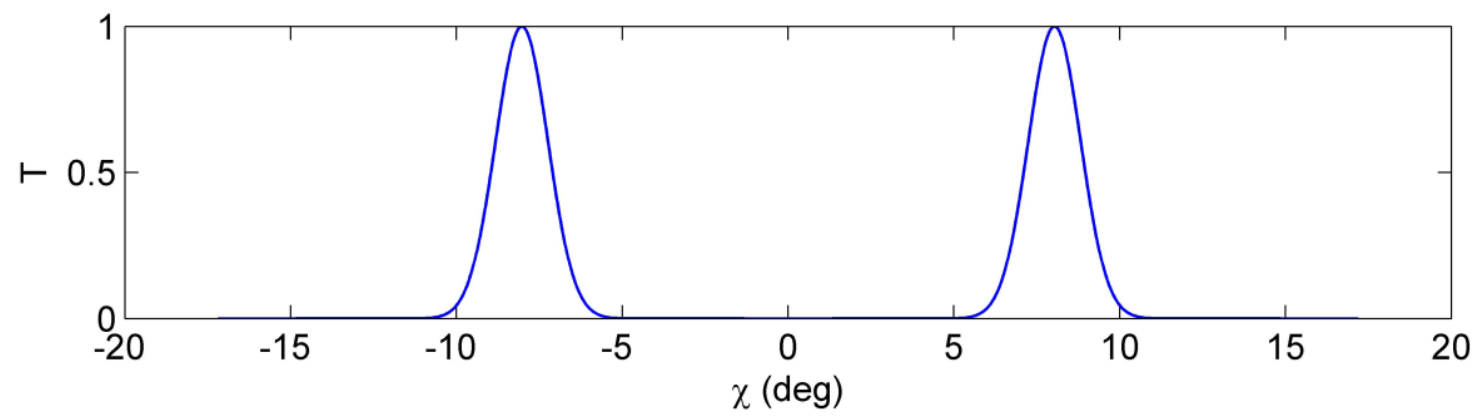

Figure 4 . The linear absorption coefficient (mode converted intensity divided by incident intensity) of the $\mathrm{O}$ mode at different angles of incidence. Significant absorption occur near the Spitze angles $\chi= \pm \chi_{c}$ with $\chi_{c}=8.04^{\circ}$. 
The absorption coefficient (mode converted intensity divided by incident intensity) of the O mode at different angles of incidence (Mjølhus 1990) is shown in Figure 4. The linear absorption is significant only for angles of incidence less than 1-2 degrees away from the Spitze angles. Therefore the linear absorption at the Spitze angle is a singular effect with little relevance to the generation of SLT described below.
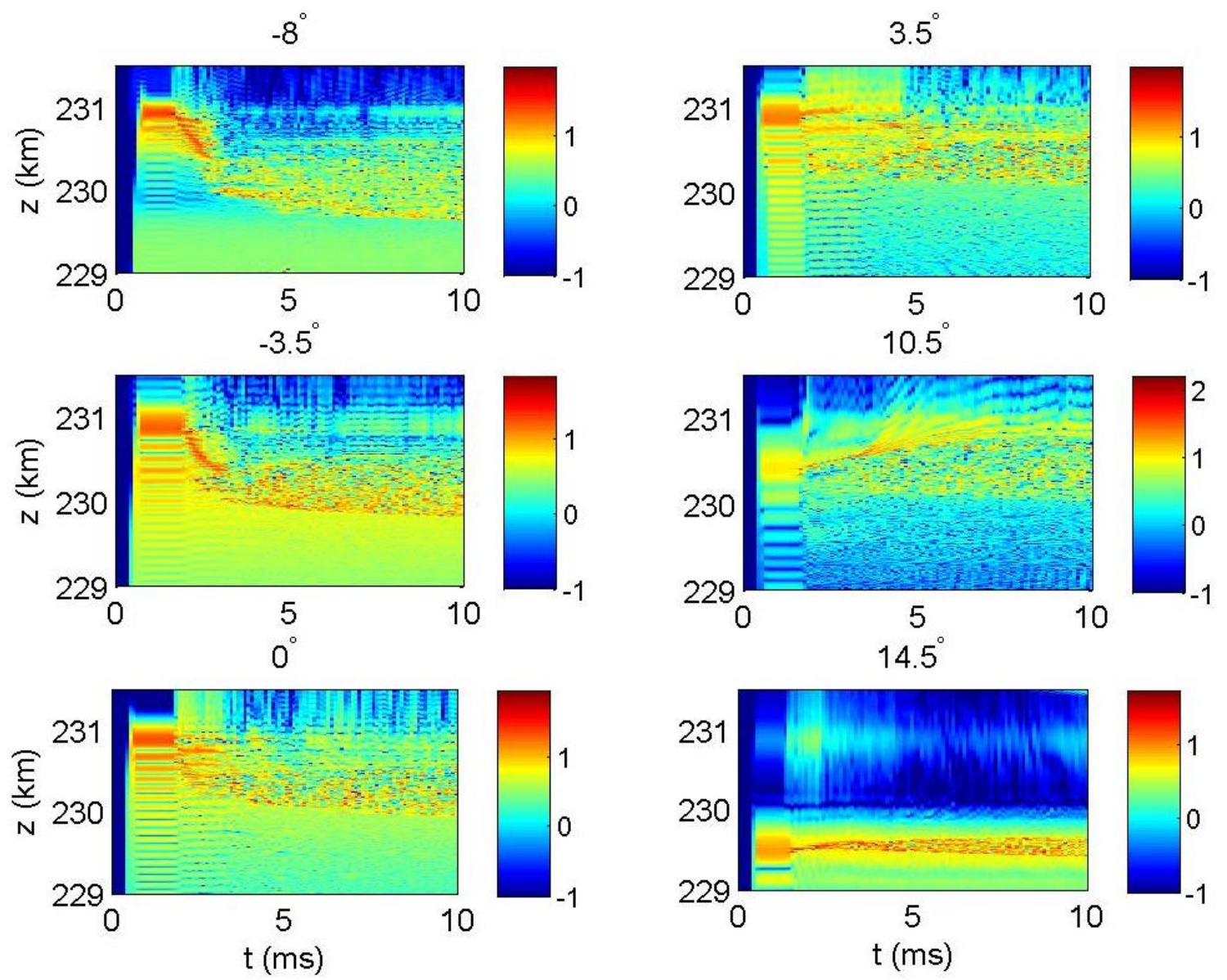

Figure 5: Time vs. altitude plot of the amplitude of the vertical electric field $\log _{10}\left(\left|E_{z}\right|\right)$ for different angles of incidence of the $\mathrm{O}$ mode wave.

The time vs. altitude development of Langmuir turbulence is illustrated in Figures 5 and 6 , which show amplitude plots of the high-frequency vertical electric field and the slowly varying ion density fluctuations. It is seen in Figure 5 that an Airy-like wave pattern of the electric field is first set up, where the first maximum near $231 \mathrm{~km}$ is just below the critical altitude for the cases $\chi=-8^{\circ}$ to $3.5^{\circ}$. At around $1.5 \mathrm{~ms}$, there is a parametric instability visible at the first maximum, and the Airy-like pattern breaks up into smallscale turbulence. This is associated with large amplitude ion density fluctuations just below the critical altitude at $231 \mathrm{~km}$ seen in Figure 6. The turbulent layer is then gradually shifted about $1 \mathrm{~km}$ downwards, while the amplitude of the electric field near the critical altitude significantly decreases. On the other hand, for $\chi=10.5^{\circ}$ and $14.5^{\circ}$, the turbulence instead begins at the first maximum of the electric field at lower altitudes, 
and then the turbulent layer (especially for $\chi=10.5^{\circ}$ ) gradually spreads to higher altitudes, as seen in both Figures 5 and 6.
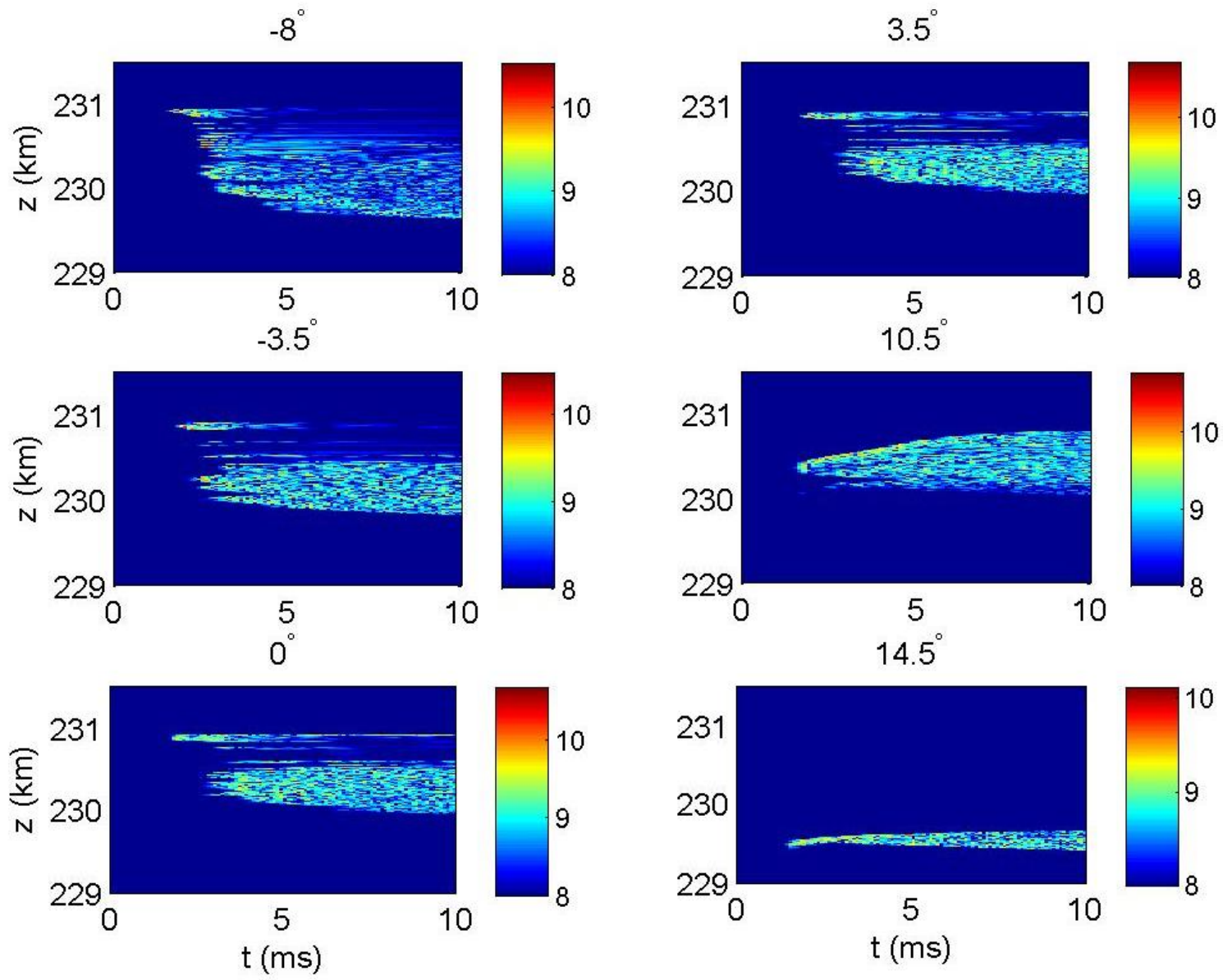

Figure 6: Time vs. altitude plot of the ion density fluctuations $\log _{10}\left(\left|n_{i 1}\right|\right)$ for different angles of incidence of the $\mathrm{O}$ mode wave. 


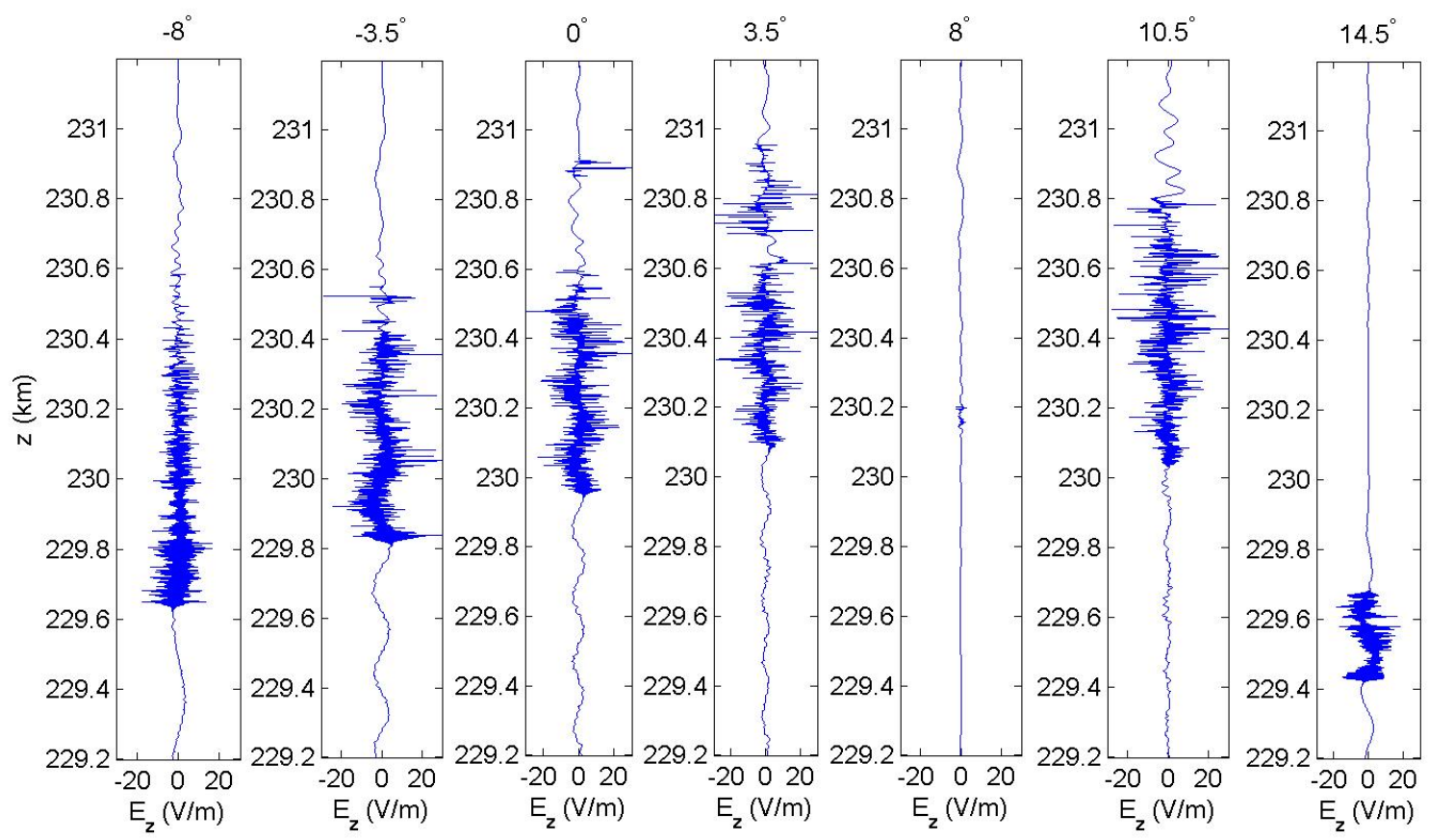

Figure 7: Summary plot of the vertical electrostatic field in the turbulent region for different angles of incidence at time $10 \mathrm{~ms}$. The case $\chi=8^{\circ}$ corresponds to the Spitze angle where the $\mathrm{O}$ mode is fully converted to $\mathrm{Z}$ mode waves and no turbulence develops. The $\mathrm{O}$ mode turning point is at $z=231.0 \mathrm{~km}$ and the upper hybrid resonance layer at $z=223.8 \mathrm{~km}$ (outside the range of the plots).

Figure 7 shows the vertical electric field at $t=10 \mathrm{~ms}$, when a quasi-steady state turbulence has developed. It is interesting to note the gradual shift to lower altitudes of the turbulence for angles away from the Spitze angle. The amplitudes of the turbulent electric fields are comparable for the for the different cases, except for the Spitze angle $\chi=8^{\circ}$, where only very week excitations are developed. At $\operatorname{MZ} \chi=14.5^{\circ}$, the electrostatic turbulence is restricted to a $200 \mathrm{~m}$ wide region near $z=229.5 \mathrm{~km}$, about 1.5 $\mathrm{km}$ below the critical layer, which corresponds to the location of the first maximum of the $\mathrm{O}$ mode wave seen in Fig. 1 for $\chi=14.5^{\circ}$. This is consistent with high resolution ion line observations by Isham et al. (1999), where the enhancements occurred several km below the critical layer outside the Spitze region. 

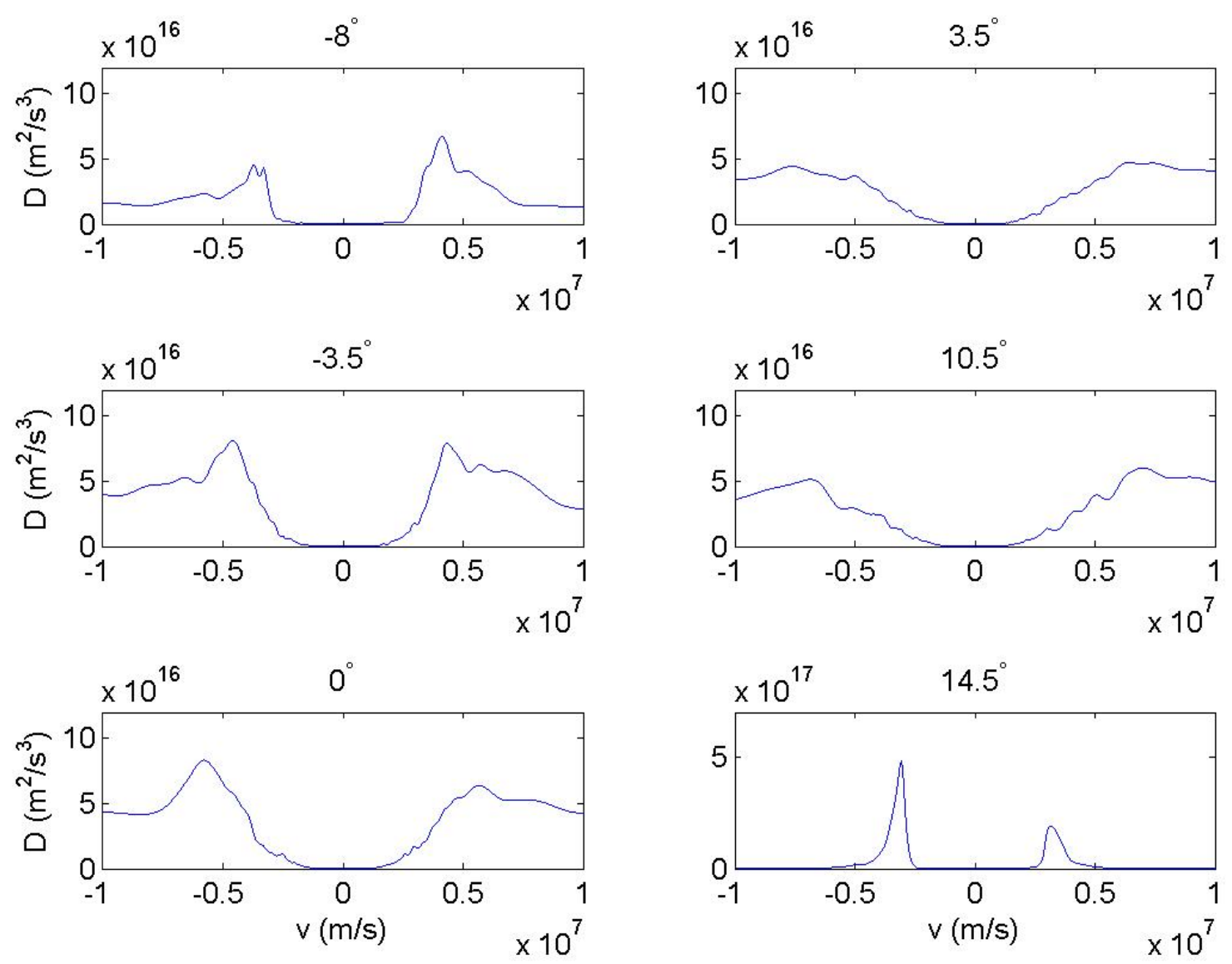

Figure 8: The diffusion coefficients in velocity space obtained from the spectra of the turbulent electric fields in Figure 7.

\section{Production of energetic electrons and formation of DAILs}

The turbulent electric fields in Figure 7 are used to calculate the acceleration of electrons for the different angles of incidence of the $\mathrm{O}$ mode wave. This process and transport of the electrons through the turbulent region can be modeled by a Fokker-Planck equation for the averaged 1D (along $\mathbf{B}_{0}$ ) electron distribution [e.g., Sagdeev and Galeev, 1969]

$$
\frac{\partial F}{\partial t}+v \frac{\partial F}{\partial z}=\frac{\partial}{\partial v} D(v) \frac{\partial F}{\partial v}
$$

with diffusion coefficient $D(v)$

$$
D(v)=\frac{\pi e^{2}}{m_{e}^{2}} \frac{W_{k}\left(\omega, \frac{\omega}{v}\right)}{|v|}
$$


where $W_{k}(\omega, \mathrm{k})=\Delta E_{z}^{2} / \Delta k$ is the spectral energy density of the electric field per wavenumber $\Delta k$, and $\Delta E^{2}$ is the differential squared electric field. Here, $W_{k}$ is in $V^{2} / m$ and normalized such that

$$
\int W_{k} d k=\frac{1}{\Delta z} \int_{z_{0}}^{z_{0}+\Delta z} E_{z}^{2} d z .
$$

Note that the integration in the right-hand side of equation (6) is over the turbulent region $z_{0}-z_{0}+\Delta z$, i.e. $229.62-230.60,229.80-230.48,229.94-230.60,230.07-$ $230.96,230.03-230.81$, and $229.42-229.69 \mathrm{~km}$ for $\chi=-8^{\circ},-3.5^{\circ}, 0^{\circ}, 3.5^{\circ}, 10.5^{\circ}$ and $14.5^{\circ}$, respectively (see figure 7). Figure 8 shows the diffusion coefficient $D(v)$ calculated by taking the Fourier transform of $E_{z}$ in Figure 7 with $\omega$ fixed at the pump frequency. Similar as in Eliasson et al. (2012), we have for numerical convenience convolved $W_{k}$ by a Gaussian to remove spikes in $D(v)$. The diffusion coefficient is significantly non-zero only at relatively large velocities. Hence only high-velocity components in the electron velocity distribution will be significantly accelerated by the SLT. Electron acceleration is favored by significant values of $D$ at small $v$. Broad coefficients (e.g. for $\chi=0^{\circ}$ and $3.5^{\circ}$ ) indicate strong Langmuir turbulence, while spiky coefficients (e.g. for $\chi=14.5^{\circ}$ ) indicate that weak turbulence (3-wave coupling) is dominant. This is consistent with previous studies by Mjølhus et al. (1995) who found that SLT is dominant near the critical layer, while at lower altitudes, the turbulence is characterized by cascading Langmuir turbulence.
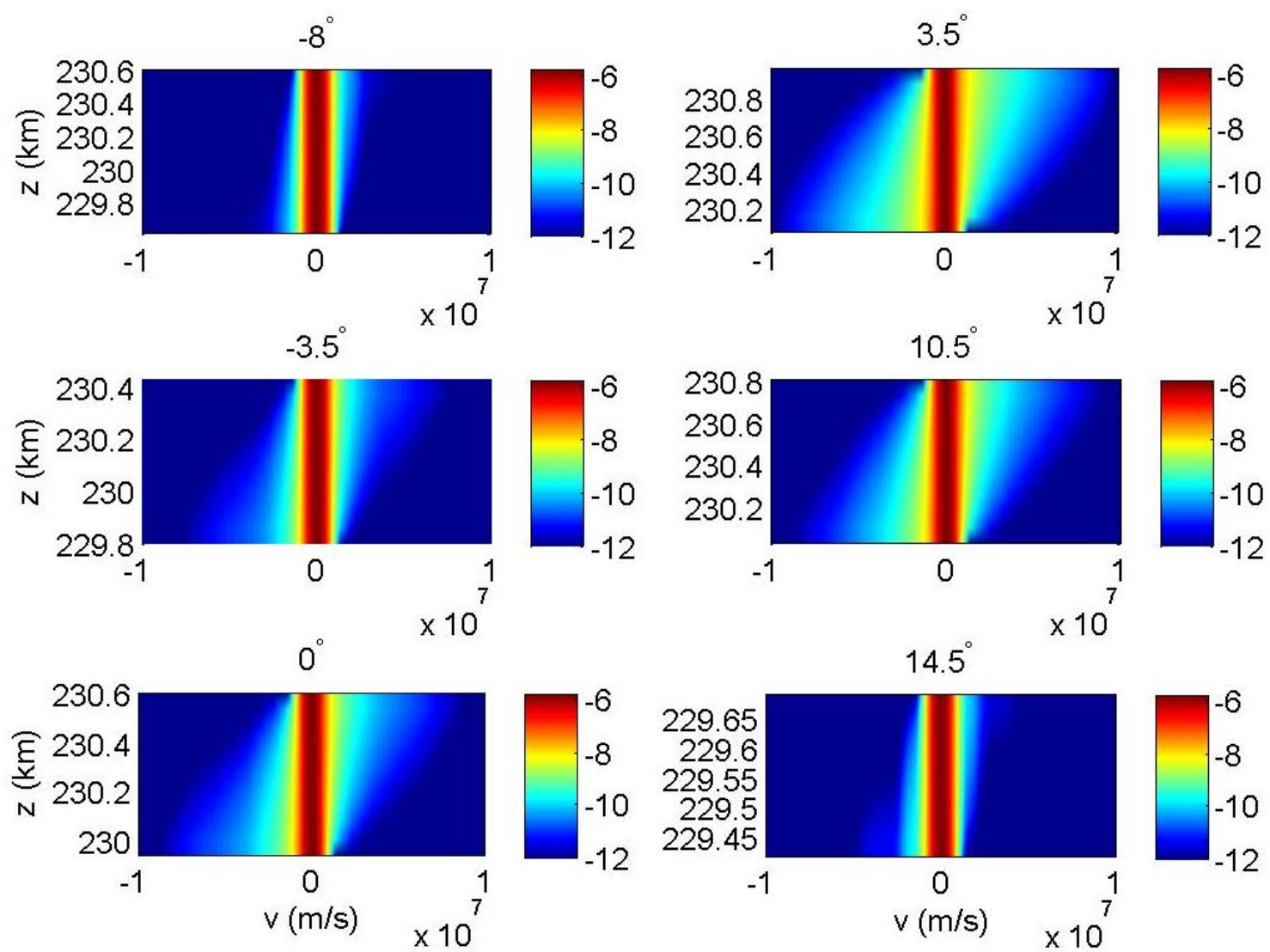

Figure 9: The electron distribution function in the heated region, 10-logarithmic scale. 
Using the diffusion coefficients in Fig. 8 as input to Fokker-Planck simulations gives the electron velocity distributions in Fig. 9. As boundary conditions we use a Maxwellian electron distribution with an electron temperature of $4000 \mathrm{~K}$ for electrons streaming into the turbulent region, while electrons on the opposite side streaming towards the boundary are allowed to reach the boundary and are removed from the simulations. The FokkerPlanck simulations are run until steady state solutions are obtained, after which the results are recorded. Significant electron diffusion in velocity is seen only for $\chi=-3.5^{\circ}$ to $10.5^{\circ}$, except for the Spitze angle case $\chi=8^{\circ}$, which is not shown. Even though for the case $\chi=14.5^{\circ}$ the diffusion coefficient has two thin peaks with an amplitude as high as $5 \times 10^{17} \mathrm{~m}^{2} / \mathrm{s}^{3}$, i.e. almost an order of magnitude higher than that for the case of $\chi=$ $3.5^{\circ}$, we see in Fig. 8 that the formation of high-velocity tails in the electron distribution function is much less significant at $\chi=14.5^{\circ}$ compared to $\chi=3.5^{\circ}$. Thus, for the acceleration of electrons by SLT, the values of the diffusion coefficient at smaller velocities are more important than its over-all maximum amplitude.

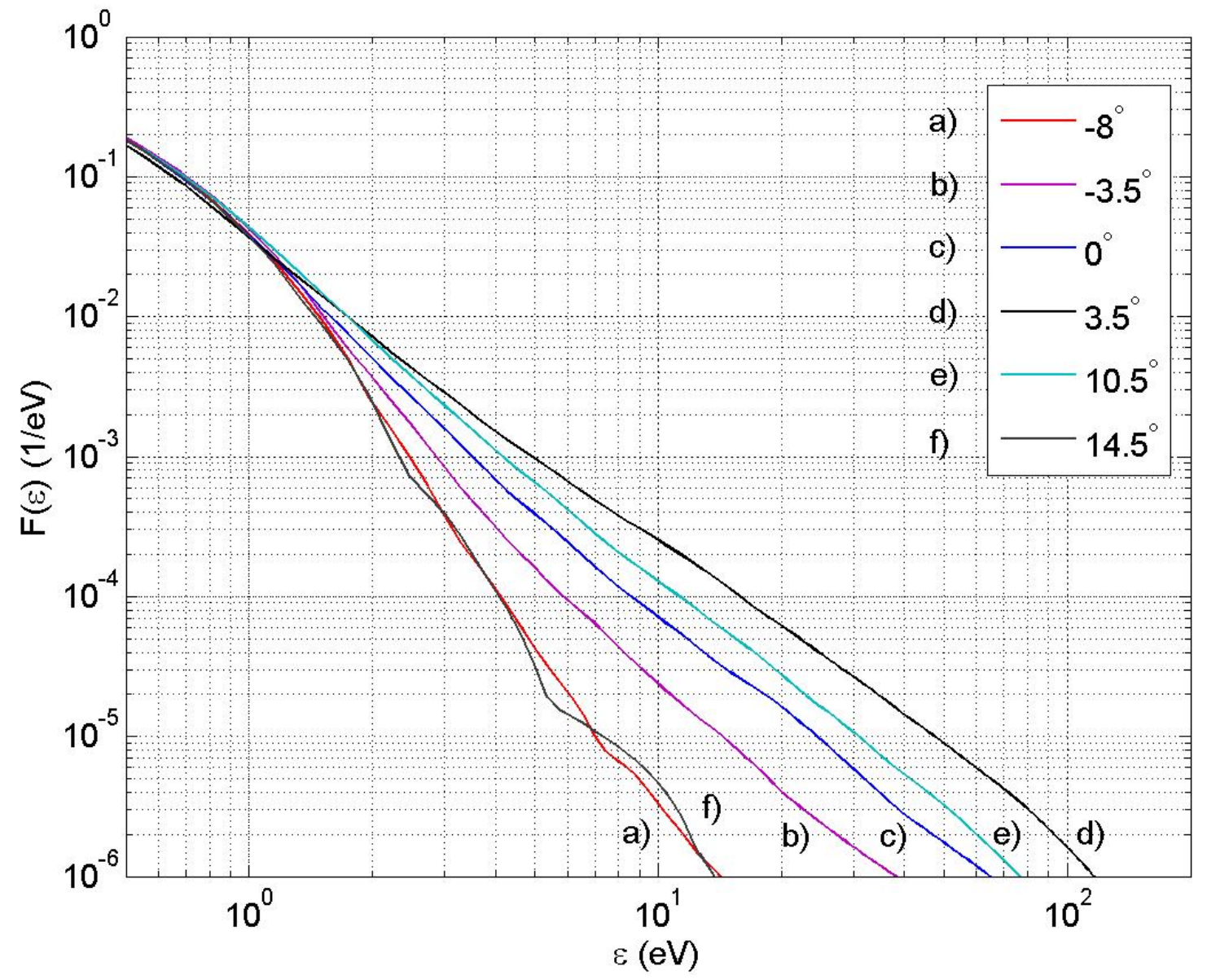

Figure 10: Energy distribution at the boundary for the different cases. Significant electron acceleration is seen for the cases $-3.5^{\circ}$ to $10.5^{\circ}$. The Spitze angle case $\chi=8^{\circ}$ gives only negligibly small electron acceleration and is therefore not shown. 
The corresponding energy distribution of the electrons exiting the turbulent region is shown in Fig. 10 for different angles of incidence of the $\mathrm{O}$ mode wave. Significant acceleration of electrons is only seen in the cases $\chi=-3.5^{\circ}$ to $10.5^{\circ}$. (We again excluded the Spitze angle $\chi=8^{\circ}$.) In particular, we see that the broadest energy tails are formed for $\chi=3.5^{\circ}$ and $10.5^{\circ}$, followed by $\chi=0^{\circ}$. We recall from Figure 7 that for $\chi=$ $3.5^{\circ}$ and $10.5^{\circ}$, the turbulence occur at higher altitudes, where strong Langmuir turbulence dominates over weak turbulence, which may contribute to the more efficient electron acceleration for these cases.
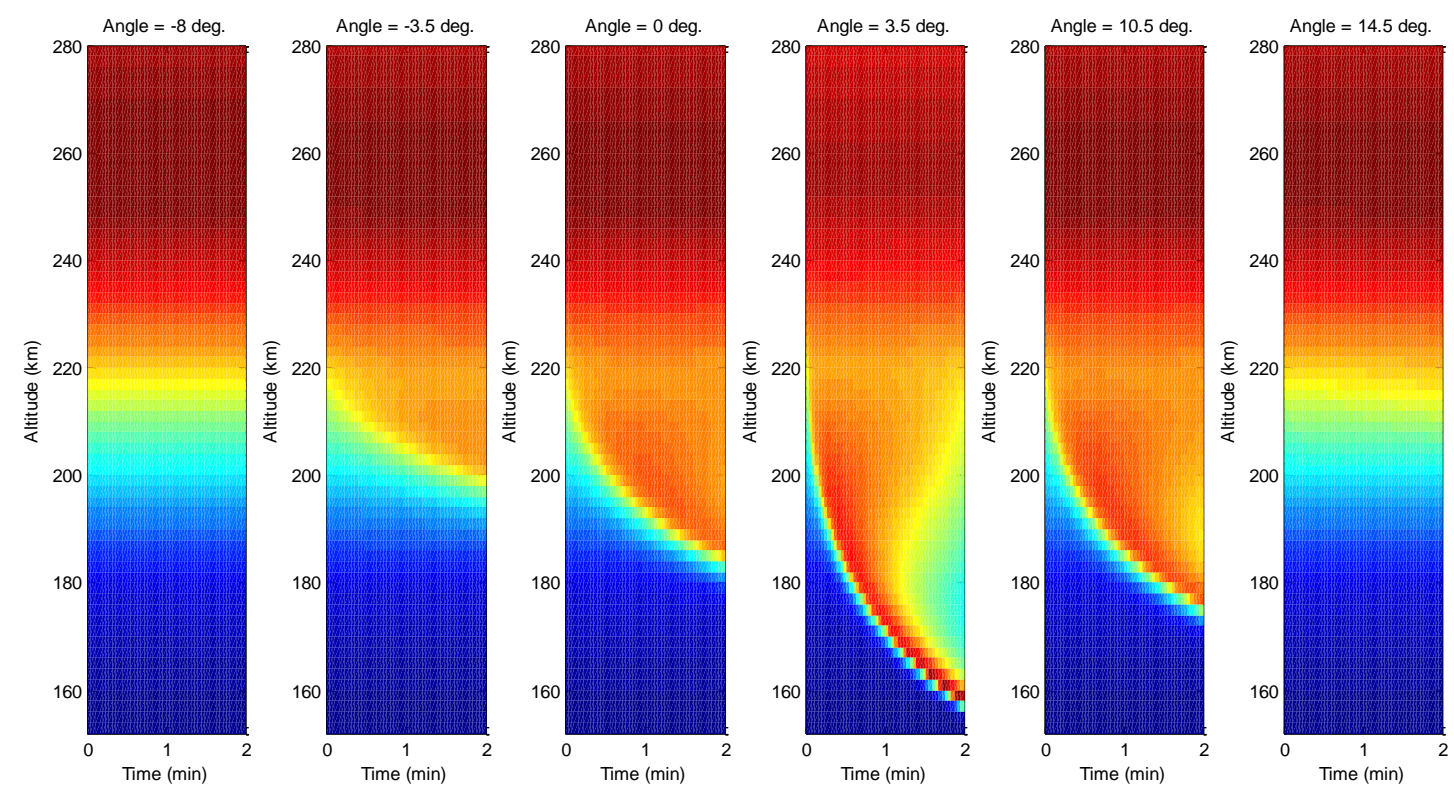

Figure 11: Altitude vs. time dependence of the electron density at different angles of incidence.

Using a kinetic transport model for the energetic electrons and a chemical kinetic model for the ionization of the neutral gas by the impact of electrons, charge exchange collisions, and recombination between electrons and molecular ions, we build a dynamic model of the formation of DAILs; see sections 3 and 4 of Eliasson et al. (2012) for details of the model. The dominant species in the neutral gas is molecular and atomic oxygen and molecular nitrogen, which can be ionized by the energetic electrons. Of these, $\mathrm{O}_{2}, \mathrm{O}$, and $\mathrm{N}_{2}$ has an ionization potential of $12.1 \mathrm{eV}, 13.61 \mathrm{eV}$ and $15.8 \mathrm{eV}$, respectively (Rees 1998). The main chemical processes in the model (Eliasson et al. 2012) are ionization of atomic and molecular oxygen and molecular nitrogen by the accelerated electrons, production of molecular oxygen ions and nitrogen monoxide ions via charge exchange collisions, e.g. $\mathrm{O}^{+}+\mathrm{O}_{2} \rightarrow \mathrm{O}_{2}^{+}+\mathrm{O}$ and $\mathrm{O}^{+}+\mathrm{N}_{2} \rightarrow \mathrm{NO}^{+}+\mathrm{N}$, and dissociative recombination between electrons and molecular ions [e.g., Schunk and Nagy, 2000]. Optical emissions include $557.7 \mathrm{~nm}, 630.0 \mathrm{~nm}$ and $777.4 \mathrm{~nm}$ from the excitation of the ${ }^{1} \mathrm{~S},{ }^{1} \mathrm{D}$ and ${ }^{5} \mathrm{P}$ states of atomic oxygen, excited at 4.19, 1.97 and 10.74 , respectively. The $427.8 \mathrm{~nm}$ optical emission from the first negative band of the $N_{2}^{+}$ion at $18.75 \mathrm{eV}$ can be used as an indicator that electrons have exceeded the ionization threshold of all major species in the neutral gas. The strong optical emissions at 557.7, 777.4, and $427.8 \mathrm{~nm}$ observed in the experiments at HAARP 
(e.g. Pedersen et al. 2009) are consistent with ionization of the neutral gas by energetic electrons. The time vs. altitude dependence of the electron density for the different angles of incidence of the $\mathrm{O}$ mode is shown in Figure 11. New plasma is created below the original reflection point of the $\mathrm{O}$ mode wave, due to the ionization of the neutral gas by energetic electrons. When the plasma density reaches the critical density of the $\mathrm{O}$ mode, the turbulent layer is shifted downwards. This gives rise to an ionization front rapidly descending from the original altitude of about $230 \mathrm{~km}$. It is interesting to note that the ionization of the neutral gas seems to be most efficient for angles of incidence $\chi=3.5^{\circ}$ and $10.5^{\circ}$, which and between the vertical and the MZ. On the other hand, no DAILs are formed when the $\mathrm{O}$ mode beam is directed along the negative Spitze, $\chi=-8^{\circ}$, or MZ, $\chi=14.5^{\circ}$. (The singular Spitze case $\chi=8^{\circ}$ is again excluded.)

a)

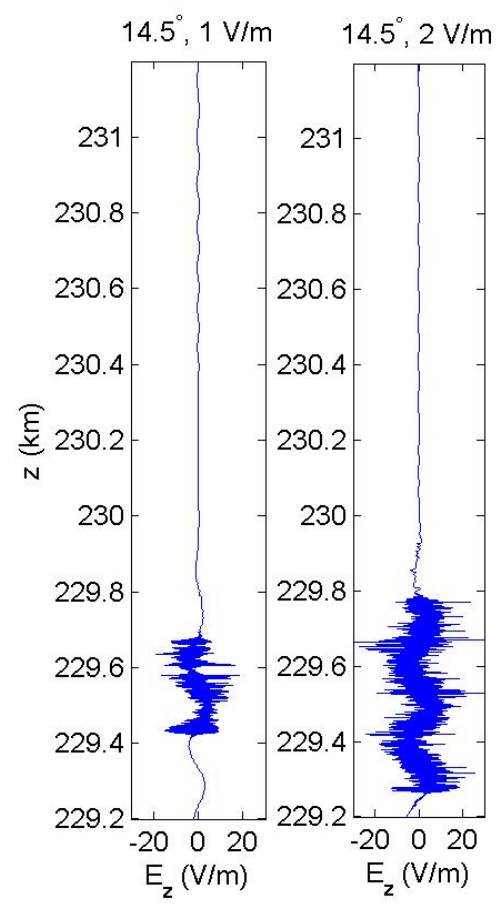

b)
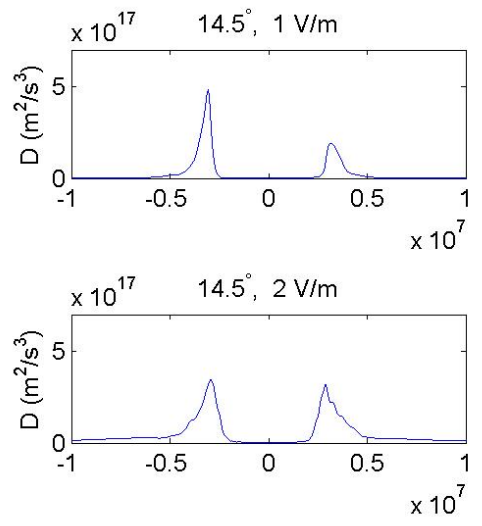

c)

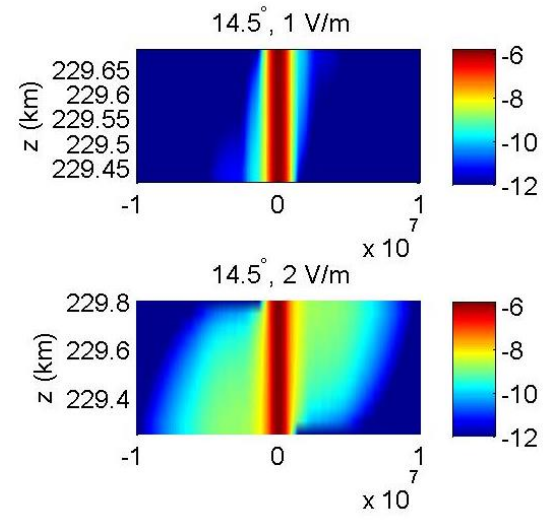

Figure 12: Comparison O mode amplitudes $1 \mathrm{~V} / \mathrm{m}$ and $2 \mathrm{~V} / \mathrm{m}$ at MZ. a) Vertical electric field $E_{z}$. b) Diffusion coefficient. c) Electron velocity distribution function.

The result that no significant electron heating is observed for the angle of incidence at MZ may appear surprising, since the experiments so far have been carried out with the heater beam along MZ, and significant electron heating and the formation of DAILs have been observed. However, our model excludes such important effects as upper hybrid turbulence and the formation of magnetic field aligned striations, and the focusing of the electromagnetic beam on striations (e.g. Gurevich et al. 2002) or due to large-scale 
thermal expansion of the plasma (Hansen et al. 1990). In the numerical modelling, we have also neglected the effects of the finite width of the heating beam. We first investigate what would be the effect of focusing of the electromagnetic beam such that its field amplitude is $2 \mathrm{~V} / \mathrm{m}$ instead of $1 \mathrm{~V} / \mathrm{m}$ when it reaches the critical region. In doing so, we carried out a simulation with the injected $\mathrm{O}$ mode amplitude $2 \mathrm{~V} / \mathrm{m}$ and compared with $1 \mathrm{~V} / \mathrm{m}$ injected $\mathrm{O}$ mode. The results are shown in Figures 12 and 13.

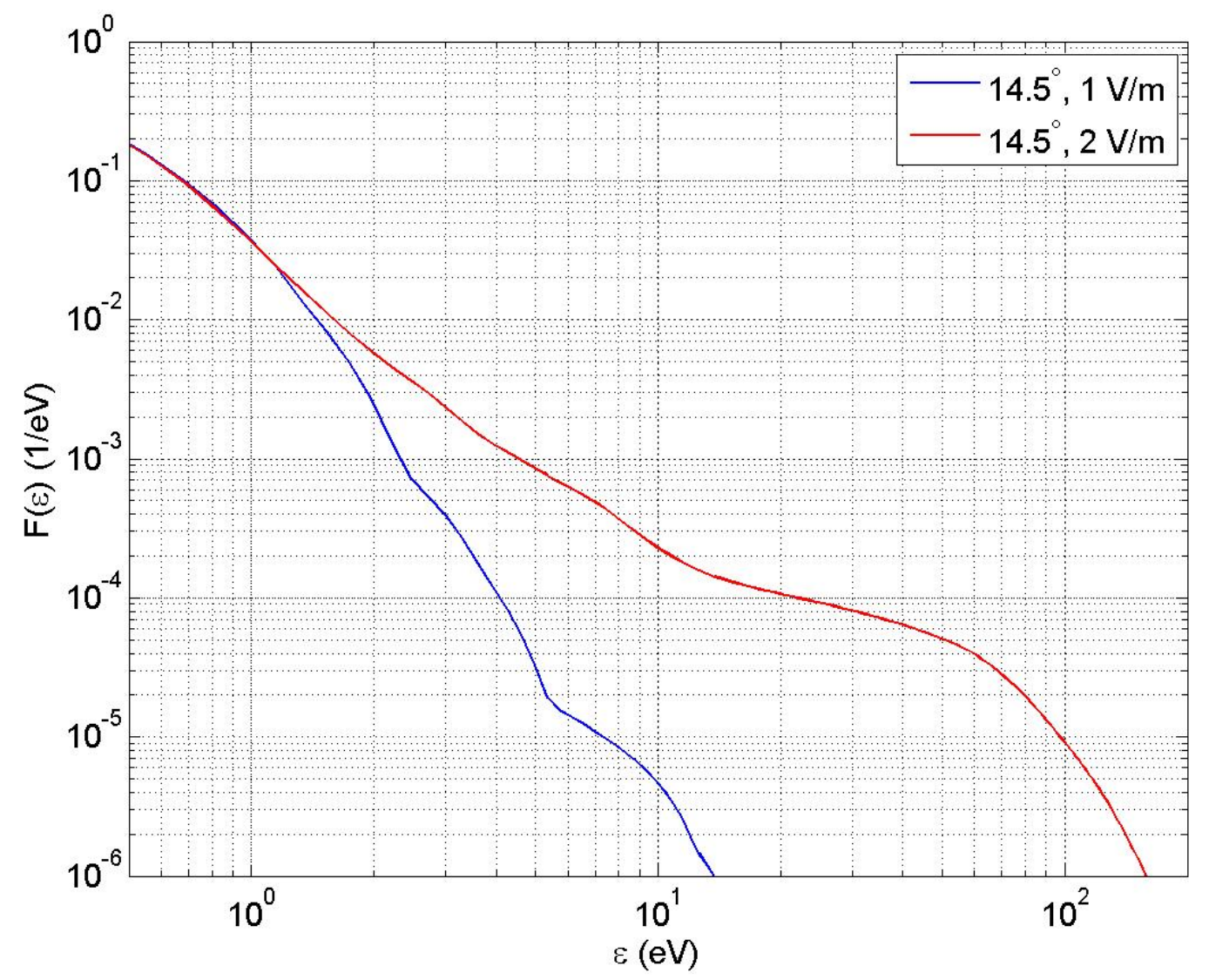

Figure 13: Electron energy distribution for $\mathrm{O}$ mode amplitudes $1 \mathrm{~V} / \mathrm{m}$ and $2 \mathrm{~V} / \mathrm{m}$ at $\mathrm{MZ}$.

The increase of the $\mathrm{O}$ mode electric field a factor 2 has a significant effect on the SLT and associated electron acceleration. As seen in Figure 12a, the turbulent region widens about factor 2, and the amplitude also increases for the larger $\mathrm{O}$ mode amplitude. More importantly, Figure $12 \mathrm{~b}$ shows that the diffusion coefficient has non-zero values for smaller velocities and therefore the SLT can interact with and resonantly accelerate a larger fraction of the electrons. This leads to a significant widening of the electron velocity distribution function, as seen in Figure 12c. The energy distribution of the electrons leaving the turbulent region, plotted in Figure 13, shows that for $2 \mathrm{~V} / \mathrm{m}$ O mode wave, the electrons have a high-energy tail at least as significant as the most favorable case $\chi=3.5^{\circ}$ in Figure 10. This would also lead to the formation of DAILs such as for the $\chi=3.5^{\circ}$ shown in Figure 11, which would also be in line with experiments (Pedersen et al. 2010; Mishin and Pedersen 2011). 

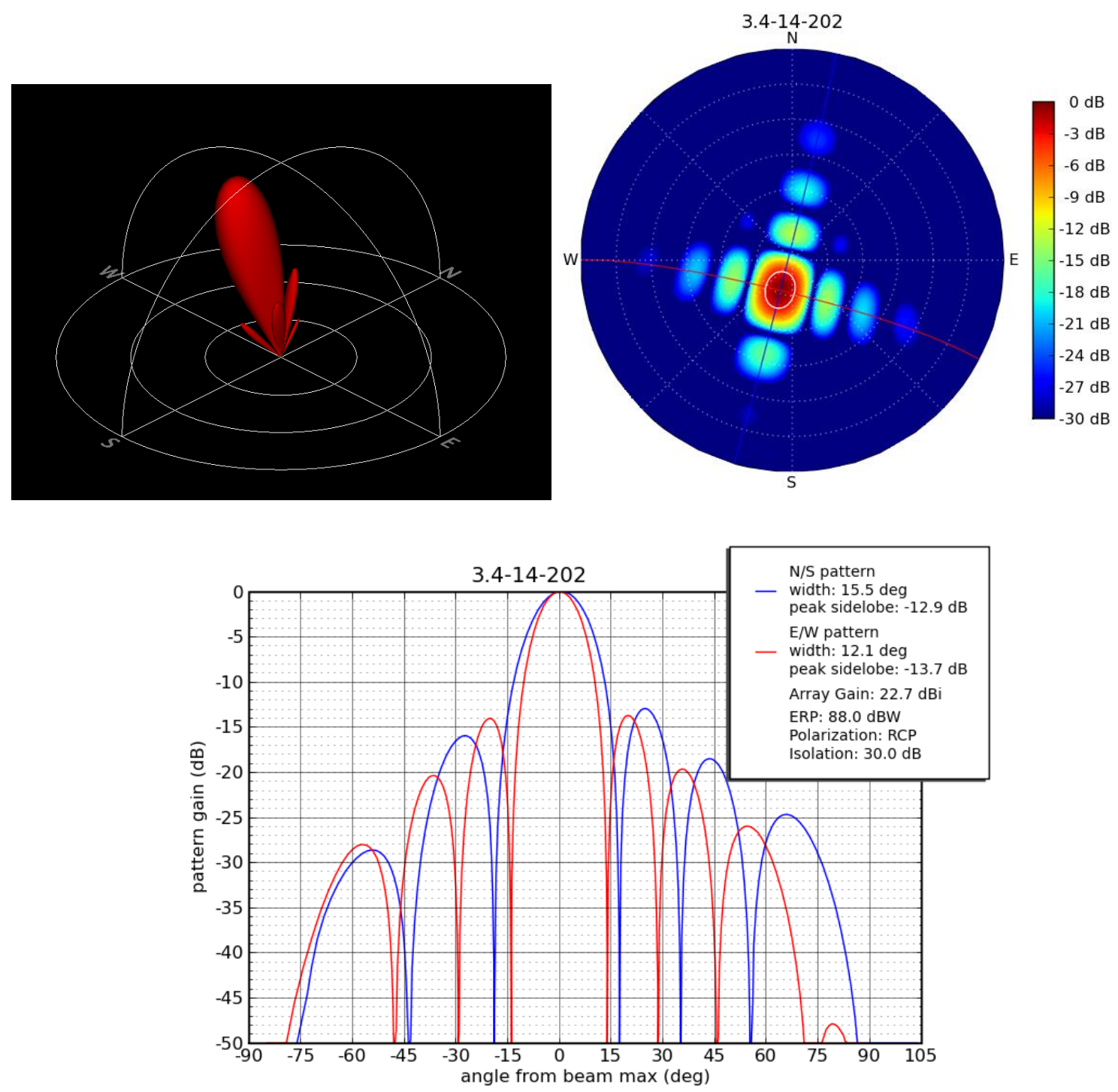

Figure 14: HAARP radiation pattern at MZ for 3.4 MHz transmitting frequency, showing that the beam's half-width is about $15.5^{\circ}$ around $\mathrm{MZ}$ in the North/South direction and $12.1^{\circ}$ in the East/West direction.

We next discuss the effects of the finite width of the heating beam at HAARP when directed at MZ. The finite beam width leads to a range of angles of incidence around the direction of the main lobe of the beam. Figure 14 shows the radiation pattern for the HAARP beam at MZ for frequency 3.4 MHz. Most of the energy is delivered within an ellipsoid around the $\mathrm{MZ}$ that includes the $3.5^{\circ}$ and $10.5^{\circ}$ regions. This effect would become even stronger if one adds propagation induced diffraction. Unless the gain is more than 40-50 dB (which holds for frequencies below $5 \mathrm{MHz}$ ) the majority of the power is delivered at angles plus or minus 5 to 8 degrees from the MZ. Therefore, SLT will be generated at angles including the $3.5^{\circ}$ and $10.5^{\circ}$ regions also when the main lobe is directed at MZ. 


\section{Discussion}

We have numerically investigated the importance of different angles of incidence of large amplitude $\mathrm{O}$ mode waves on the development of ionospheric strong Langmuir turbulence (SLT) during ionospheric modification experiments at HAARP. The ionospheric parameters are compatible also for other northern hemisphere facilities. The SLT near the critical layer of the $\mathrm{O}$ mode wave leads to the acceleration of electrons and the formation of artificial ionospheric layers. This is attributed to the ionization of the neutral gas by energetic electrons accelerated by the SLT. For angles of incidence within the Spitze angle, turbulence first develops at the first maximum of the Airy-like standing wave pattern just below the critical layer of the $\mathrm{O}$ mode, and after a few milliseconds the turbulent layer shifts about one kilometer downwards. For angles outside the Spitze region, the $\mathrm{O}$ mode wave is reflected at a lower altitude where the turbulence develops. Most efficient electron acceleration and ionization is obtained at angles of incidence between MZ and the vertical, where SLT dominates over weak turbulence. However at $\mathrm{MZ}$ (14.5 ${ }^{\circ}$ to the vertical for HAARP), the turbulence takes place at an altitude about 1.5 $\mathrm{km}$ below the critical layer, due to the turning of the $\mathrm{O}$ mode at lower altitudes outside the Spitze region. The results that the SLT is enhanced and takes place below the critical layer at angles between the Spitze and MZ are consistent with radar observations of Langmuir turbulence during low duty high-frequency ionospheric interaction experiments (Isham et al. 1999). The turbulence at MZ leads to a narrowly peaked diffusion coefficient a large velocities, and as a result less efficient electron acceleration by the turbulence. However, the present model excludes the effects of focusing of the electromagnetic beam on striations (e.g. Gurevich et al. 2002) and due to large-scale thermal expansion of the plasma (Hansen et al. 1990). Simulating the focusing effects by increasing the $\mathrm{O}$ mode amplitude a factor 2 indeed shows a dramatic effect on the SLT and resulting electron acceleration, leading to a significant formation of high-energy tails in the electron energy distribution. In addition to the focusing effects, the heating beam at HAARP also delivers most of its energy at an ellipsoid that includes the regions near $3.5^{\circ}$ and $10.5^{\circ}$ where SLT can be efficiently generated. The combined effects of propagation induced beam focusing and the finite width of the transmitted beam may explain the efficient generation of DAILs when the HAARP heater beam is directed along MZ (Pedersen et al. 2010, Mishin and Pedersen 2011). Future modeling should include the effects of upper hybrid turbulence in striations, self-focusing effects and anomalous absorption of the electromagnetic beam, and the resulting electron heating in a selfconsistent manner.

\section{Acknowledgment}

This work was supported by DARPA via a subcontract N684228 with BAE Systems and also by the MURI grants N000140710789 and FA95501410019. EVM was supported by the Air Force Office of Scientific Research. 


\section{References}

Eliasson, B. (2013), Full-scale simulations of ionospheric Langmuir turbulence, Mod. Phys. Lett. B, 27(8), 1330005, doi:10.1142/S0217984913300056.

Eliasson, B., X. Shao, G. Milikh, E. V. Mishin, and K. Papadopoulos (2012), Numerical modeling of artificial ionospheric layers driven by high-power HF-heating, J. Geophys. Res., 117, A10321, doi:10.1029/2012JA018105.

Gurevich, A. V., K. P. Zybin, H. C. Carlson, and T. Pedersen, Magnetic zenith effect in ionospheric modifications, Phys. Lett. A 305, 264-274 (2002).

Hansen, J. D., G. J. Morales, L. M. Duncan, J. E. Maggs, and G. Dimonte (1990), Largescale ionospheric modifications produced by nonlinear refraction of an hf wave, Phys. Rev. Lett., 65, 3285.

Honary, F., N. Borisov, M. Beharrell, and A. Senior (2011), Temporal development of the magnetic zenith effect, J. Geophys. Res., 116, A06309.

Isham, B., M. T. Rietveld, T. Hagfors, C. La Hoz, E. Mishin, W. Kofman, T. B. Leyser, A. P. van Eyken (1999), Aspect angle dependence of HF enhanced incoherent backscatter, Adv. Space Res. 24(8), 1003-1006, doi:10.1016/S0273-1177(99)00555-4.

Mishin, E., and T. Pedersen (2011), Ionizing wave via high-power HF acceleration, Geophys. Res. Lett., 38, L01105, doi:10.1029/2010GL046045.

Mjølhus, E. (1990), On linear mode conversion in a magnetized plasma, Radio Sci., 25(6), 1321-1339.

Mjølhus, E., A. Hanssen, and D. F. DuBois (1995), Radiation from electromagnetically driven Langmuir turbulence, J. Geophys. Res., 100(A9), 17,527-17,541, doi:10.1029/95JA01158.

Mjølhus, E., E. Helmersen, and D. F. DuBois (2003), Geometric aspects of HF driven Langmuir turbulence in the ionosphere, Nonlin. Proc. Geophys., 10, 151-177, doi:10.5194/npg-10-151-2003.

Pedersen, T., B. Gustavsson, E. Mishin, E. MacKenzie, H. C. Carlson, M. Starks, and T. Mills (2009), Optical ring formation and ionization production in high-power HF heating experiments at HAARP, Geophys. Res. Lett., 36, L18107, doi:10.1029/2009GL040047.

Pedersen, T., B. Gustavsson, E. Mishin, E. Kendall, T. Mills, H. C. Carlson, and A. L. Snyder (2010), Creation of artificial ionospheric layers using high-power HF waves, Geophys. Res. Lett., 37, L02106, doi:10.1029/2009GL041895. 
Rees, M. H. (1989), Physics and Chemistry of the Upper Atmosphere (Cambridge University Press, Cambridge, U.K.)

Sagdeev, R., and A. Galeev (1969), Nonlinear Plasma Theory, Benjamin, New York.

Sergeev, E., S. Grach, A. Shindin, E. Mishin, P. Bernhardt, S. Briczinski, B. Isham, M. Broughton, J. LaBelle, and B. Watkins (2013), Artificial ionospheric layers during pump frequency stepping near the 4th gyroharmonic at HAARP, Phys. Rev. Lett., 110, 065002, doi:10.1103/PhysRevLett.110.065002.

Schunk, R. W., and A. N. Nagy (2000), Ionospheres - Physics, Plasma Physics and Chemistry, (Cambridge University Press, Cambridge, U.K.).

Stix, T. H. (1992) Waves in Plasmas (American Institute of Physics, New York).

Whitham, G. B. (1974), Linear and Nonlinear Waves (John Wiley \& Sons, Inc., New York).

Zakharov, V. E. (1972), Collapse of Langmuir Waves, Soviet J. Exp. Theor. Phys. (JETP), 39, 908. 